\title{
Disordered driven coupled cavity arrays: Nonequilibrium stochastic mean-field theory
}

\author{
Gytis Kulaitis, ${ }^{1}$ Frank Krüger, ${ }^{1}$ Felix Nissen, ${ }^{2}$ and Jonathan Keeling ${ }^{1}$ \\ ${ }^{1}$ Scottish Universities Physics Alliance, School of Physics and Astronomy, University of St Andrews, \\ St Andrews KY16 9SS, United Kingdom \\ ${ }^{2}$ Cavendish Laboratory, University of Cambridge, Cambridge CB3 OHE, United Kingdom
}

(Received 21 December 2012; published 29 January 2013)

\begin{abstract}
We study the interplay of disorder with pumping and decay in coupled qubit-cavity arrays, the JaynesCummings-Hubbard model. We find that relatively weak disorder can wash out the bistability present in the clean pumped system and that moreover the combination of disorder in on-site energies and decay can lead to effective phase disorder. To explore these questions, we present a nonequilibrium generalization of stochastic mean-field theory, providing a simple tool to address such questions. This technique is developed for rather general forms of light-matter coupling, driving, dissipation, and on-site disorder, making it applicable to a wide range of systems.
\end{abstract}

DOI: $10.1103 /$ PhysRevA.87.013840

PACS number(s): 42.50.Pq, 72.15.Rn

\section{INTRODUCTION}

Quantum simulation [1] concerns how controllable quantum systems can be used to model particular desirable Hamiltonians in order to find the ground state, or other properties, of otherwise hard-to-simulate problems. Recently, there has been significant progress in realizing quantum emulators based on systems including ultracold atoms [2], Rydberg atoms [3], trapped ions $[4,5]$, and superconducting qubits in microwave cavities [6-8]. One approach that has been used recently for cold atoms is to engineer an effective Hamiltonian in a rotating frame by using a Raman pumping scheme [9]. This approach has been used to realize the super-radiant transition in the Dicke model [10]. In such cases, and more generally for coupled matter-light systems such as superconducting qubits in microwave cavities, it can be crucially important to understand the effects of losses and dissipation. For example, in the Dicke model, the presence of losses means the critical behavior [11-13] becomes classical [14] due to the effective temperature introduced by losses. Similar issues generally can be expected to occur in any open driven system, and this therefore may have consequences across the range of experimental systems considered as potential quantum emulators, and in particular, for coupled light-matter systems [15].

Another model where quantum simulation has been explored [16] is the disordered Bose-Hubbard model (BHM) [17]. This model consists of bosonic particles hopping between sites with repulsion between particles on the same lattice site. This model can be simulated with ultracold bosonic atoms, introducing disorder in a highly controlled manner by superimposing a fine-grained optical speckle potential with a periodic optical lattice [18-21]. In the presence of weak disorder in the on-site energies, three possible ground states exist at zero temperature: a superfluid phase and two insulating phases. The two insulating phases are the incompressible Mott insulator and the compressible Bose glass. In the Mott insulator, the particles are localized because of strong local repulsions; in the Bose glass, particles are localized because of the disorder potential. Despite the long history of the BHM, it is only recently that several aspects of this model have been fully understood, such as confirmation that the Bose-glass phase always intervenes between Mott insulator and superfluid [22] and the distinction between the Mott insulator and Bose glass regarding whether fluctuations are self-averaging [23]. Even if quantum simulation of such a model with an effective Hamiltonian in a dissipative system can model only the finite temperature case, this may of itself be enough to answer questions such as whether the finite-temperature insulating phase is self-averaging. However, as we discuss further below, disorder and nonequilibrium effects can conspire to significantly change the behavior (and universality class) of the model system.

The Jaynes-Cummings-Hubbard model (JCHM) [24] is very closely related [6] to the BHM and can be more directly realized in coupled cavity arrays $[6-8,15,25]$. This model naturally describes superconducting qubits in microwave cavities. The JCHM consists of photons coupled to two-level systems, considering photons confined in an array of coupled cavities, with weak hopping between different cavities. The JCHM requires that only the energy-preserving term $\left(a \sigma^{+}+a^{\dagger} \sigma^{-}\right)$ in qubit-cavity coupling is important. When counter-rotating terms $\left(a \sigma^{-}+a^{\dagger} \sigma^{+}\right)$are also important, the model is known as the Rabi-Hubbard model, the symmetry of the Hamiltonian is lowered from $\mathrm{U}(1)$ to $Z_{2}$, and the phase diagram significantly changes [26]. In the case of the JCHM, previous work has shown how, in equilibrium, including on-site disorder leads to behavior very similar to the BHM [27], as may be expected from the symmetries of the problem [6].

In this paper we study the nonequilibrium JCHM in the presence of disorder. We focus on the simplest possible form of pumping and decay, i.e., uniform coherent pumping, as has previously been studied in the clean limit [28-30]. In this case, all symmetries are broken by the pumping, and no phase transitions are expected. Nonetheless, the behavior we observe and discuss for this case clearly shows how new physics would also arise with other forms of pumping which need not break the symmetries of the model. In particular, we see that pumping and dissipation can transform on-site energy disorder into phase disorder, destroying long-range order in the superfluid phase. In addition, we explore the fate of the bistability seen in the clean nonequilibrium JCHM [28].

To explore these questions, we use a generalized "stochastic" mean-field theory [31-33], which involves selfconsistency equations for the probability distribution of local order parameters. We extend this approach to apply to open 
quantum systems. Such an approach is approximate and only becomes well controlled at high coordination number (i.e., in high dimensions). Nonetheless, it provides a simple tool to effectively explore the interplay of disorder and pumping and to see whether effective Hamiltonians for open systems could in principle be used to simulate disordered quantum systems.

The remainder of this paper is arranged as follows. In Sec. II we generalize stochastic mean-field theory (SMFT) to treat disorder in open quantum systems. The technique is introduced for rather general forms of pumping, decay, and on-site disorder. As an example, we apply the SMFT to the dissipative JCHM. In Sec. III, we first briefly summarize the behavior of the JCHM in the absence of disorder and then discuss the effects of on-site disorder in the excitation energies of the two-level systems.

\section{STOCHASTIC MEAN FIELD THEORY OF OPEN SYSTEMS}

This section briefly summarizes the SMFT approach as applied to the nonequilibrium problem. The equilibrium SMFT was introduced in the context of disordered antiferromagnets [31] and later applied to the BHM [32,33], and it has more recently been applied to the JCHM [27]. We present the following discussion for a general coupled cavity array problem and apply it to the JCHM in Sec. III.

We consider an array of cavities with coordination number $z$ and hopping $J / z$ of photons between neighboring cavities, given by the Hamiltonian

$$
H=\sum_{i} h_{i}-\frac{J}{z} \sum_{\langle i j\rangle} a_{i}^{\dagger} a_{j}
$$

where $a_{i}^{\dagger}\left(a_{i}\right)$ creates (annihilates) a photon on the $i$ th cavity. The on-site Hamiltonian $h_{i}=h\left(a_{i}, X_{i}^{(\alpha)}, \epsilon_{i}\right)$ for the individual cavities can be completely general at this point. The operators $X^{(\alpha)}$ act on the Hilbert space of the possible quantum states of the matter contained in the cavities. In the simplest cases, including the JCHM and the Rabi-Hubbard model, this will be a two-level system and the $X$ operators are spin- $\frac{1}{2}$ operators. The on-site Hamiltonian will contain a coupling between the photons and the matter degrees of freedom as well as any coherent pumping terms.

We further introduce on-site disorder $\epsilon_{i}$, which can couple either to the photon energy or to the matter in the cavity. The disorder follows a probability distribution $p(\epsilon)$ and is assumed to be uncorrelated between different cavities. For such on-site disorder the method is as developed in Refs. [32,33]. If one instead considered disorder in the hopping between sites, the problem is analogous to that originally considered in Ref. [31].

Dissipation is included on the level of a master equation for the time evolution of the density operator,

$$
\frac{d \rho}{d t}=-i[H, \rho]+\sum_{i}\left\{\frac{\kappa}{2} \mathcal{L}\left[a_{i}\right]+\sum_{\alpha} \frac{\gamma_{\alpha}}{2} \mathcal{L}\left[X_{i}^{(\alpha)}\right]\right\},
$$

where $\mathcal{L}[X]=2 X \rho X^{\dagger}-\left\{X^{\dagger} X, \rho\right\}$ denote the standard Lindblad operators.

The basic idea of SMFT is to consider a self-consistency condition for the probability distribution $P(\psi)$ of on-site coherent fields $\psi=\langle a\rangle$. From this one may find the distribution of sums of fields from neighboring sites:

$$
Q(\phi)=\int \prod d \psi_{i} \delta\left(\phi-\sum_{i} \psi_{i}\right) P\left(\psi_{i}\right),
$$

where the product and sum run over the $z$ nearest neighbors. The relation between $P$ and $Q$ simplifies in Fourier space, defined as:

$$
\tilde{Q}(\xi)=\int d \phi Q(\phi) e^{i \xi \phi}, \quad Q(\phi)=\int \frac{d \xi}{2 \pi} \tilde{Q}(\xi) e^{-i \xi \phi}
$$

for the $Q$ distribution. Using the convolution theorem we obtain $\tilde{Q}(\xi)=\tilde{P}(\xi)^{z}$.

Given the distribution of fields from neighboring sites, the self-consistency condition comes from assuming that this distribution of fields is uncorrelated with the site energies, and so one may write

$$
P(\psi)=\int d \phi \int d \epsilon Q(\phi) p(\epsilon) \delta(\psi-\lambda(\phi, \epsilon)) .
$$

Here $\lambda(\epsilon, \phi)$ gives the expectation for $\psi$ corresponding to a field $\phi$ from the neighbors and on-site energy $\epsilon$. In our case this corresponds to finding the steady-state on-site density matrix from

$$
\begin{aligned}
& \frac{d \rho_{i}}{d t}=-i\left[h_{i}^{\mathrm{eff}}, \rho_{i}\right]+\frac{\kappa}{2} \mathcal{L}\left[a_{i}\right]+\sum_{\alpha} \frac{\gamma_{\alpha}}{2} \mathcal{L}\left[X_{i}^{(\alpha)}\right] \\
& h_{i}^{\mathrm{eff}}=h_{i}-\frac{J}{z}\left(\phi a_{i}^{\dagger}+\phi^{*} a_{i}\right)
\end{aligned}
$$

and determining the expectation value $\lambda\left(\phi, \epsilon_{i}\right)=\operatorname{Tr}\left(a_{i} \rho_{i}\right)$. In steady state, $d \rho_{i} / d t=0$, the master equation for the on-site density operator (6) turns into a set of coupled linear equations for the matrix elements $\left(\rho_{i}\right)_{m n}=\left\langle m\left|\rho_{i}\right| n\right\rangle$ with respect to a basis of the product Hilbert space of the matter and photon systems. While the former is usually finite, we truncate the bosonic Hilbert space at a certain maximum number of photons per cavity.

As noted above, even for real $\phi$, the values of $\psi$ will be complex. This means it is necessary to allow for the distributions $P$ and $Q$ to extend over complex fields. Convolution of two-dimensional distributions follows as before, but with $\phi \rightarrow\left(\phi^{\prime}, \phi^{\prime \prime}\right)$ in order to use the convolution theorem. To find the steady-state solution of Eqs. (3) and (5) one may choose an initial guess for $P(\psi)$ and then iteratively update $Q(\phi), P(\psi)$ in turn. Rather than calculating the steady-state value $\lambda(\phi, \epsilon)$ for each iteration, it is more efficient to calculate an interpolated approximation to $\lambda(\phi, \epsilon)$ in advance and then use this in updating $P(\psi)$ following Eq. (5).

One may also note that it is not guaranteed that the above iteration procedure should converge nor that it should converge to an unique solution-as discussed below, the mean field decoupling introduces the possibility of multistability. However, in cases where it does converge, the solution found can be regarded as an approximate description of a possible asymptotic state of the system. When multiple solutions exist, further work is required to determine which solution is reached from given initial conditions and the rate of tunnelling events that may switch between solutions. This is discussed further in Sec. III A. In the cases presented in this paper, only one asymptotic state was found. 


\section{APPLICATION TO THE PUMPED DISSIPATIVE JCHM}

As a simple application of the above technique, and the simplest kind of pumped-dissipative array, we consider here the coherently pumped Jaynes-Cummings-Hubbard model, as studied previously in Refs. [28-30]. In terms of the general lattice problem described in Eq. (1), the JaynesCummings-Hubbard model that we consider has an on-site Hamiltonian:

$$
h_{i}=J a_{i}^{\dagger} a_{i}+\frac{\epsilon_{i}}{2} \sigma_{i}^{z}+g\left(\sigma_{i}^{+} a_{i}+\text { H.c. }\right)+f\left(a_{i} e^{i \omega_{p} t}+\text { H.c. }\right) \text {, }
$$

where $a_{i}^{\dagger}$ creates a photon in the $i$ th cavity and the spin- $\frac{1}{2}$ operators $\sigma_{i}^{+}, \sigma_{i}^{-}$describe transitions of the state of the two-level (artificial) atom on site $i$. $f$ denotes the strength of the pumping at frequency $\omega_{p}$. The cavity photon energy $J$ is chosen so that for $g=0$, the bottom of the photon dispersion is at zero energy. Disorder is introduced by considering a Gaussian distribution of $\epsilon_{i}$ of width $\sigma_{\epsilon}$, and we take the mean value $\bar{\epsilon}=0$, so that the mean detuning is as in Ref. [28]. Further, we consider loss terms of the form $\sum_{i}\left\{(\kappa / 2) \mathcal{L}\left[a_{i}\right]+(\gamma / 2) \mathcal{L}\left[\sigma_{i}^{-}\right]\right\}$. The problem can be trivially made time independent by the unitary transform $a \rightarrow a e^{-i \omega_{p} t}, \sigma^{-} \rightarrow \sigma^{-} e^{-i \omega_{p} t}$.

Other than the coherent pumping term, the problem we consider has a U(1) symmetry, and this can be used to simplify the calculation of $\lambda(\phi, \epsilon)$ as discussed in the previous section. The effective on-site problem of the JCHM has a master equation with

$$
\begin{aligned}
h_{i}^{\mathrm{eff}}= & \left(J-\omega_{p}\right) a_{i}^{\dagger} a_{i}+\frac{\epsilon_{i}-\omega_{p}}{2} \sigma_{i}^{z}+g\left(\sigma_{i}^{+} a_{i}+\text { H.c. }\right) \\
& +\left[\left(f-\frac{J \phi^{*}}{z}\right) a_{i}+\text { H.c. }\right] .
\end{aligned}
$$

One may then write the steady-state expectation $\operatorname{Tr}\left(\rho a_{i}\right)$ arising from this effective Hamiltonian along with the Lindblad terms in the form:

$$
\operatorname{Tr}(a \rho)=\lambda\left(f^{\mathrm{eff}} \equiv f-\frac{J \phi}{z}, \epsilon\right),
$$

where the last line of Eq. (8) can be written as ... [( $\left.f^{\text {eff }}\right)^{*} a_{i}+$ $f^{\text {eff }} a_{i}^{\dagger}$ ], combining both the explicit pump and the field coming from the neighboring cavities into $f^{\text {eff }}$. The advantage of writing the expression in this form is that one may note that $\lambda\left(f^{\text {eff }}, \epsilon_{i}\right)=\left(f^{\text {eff }} /\left|f^{\text {eff }}\right|\right) \lambda\left(\left|f^{\text {eff }}\right|, \epsilon_{i}\right)$; i.e., the phase of the input and output are directly related, although not equal. Thus the interpolated approximation of $\lambda$ discsussed above need only be calculated as a function of $\left|f^{\text {eff }}\right|$ and $\epsilon_{i}$.

\section{A. Summary of clean JCHM}

For comparison, we briefly summarize here the behavior in the absence of disorder. In the absence of hopping, the problem is identical to that studied by Bishop et al. [34]: The coupled qubit-cavity system has an anharmonic polariton spectrum, and so at low pumping, one can consider the response to pumping an effective two-level system. If one considers the coherent field amplitude $|\langle a\rangle|$ as a function of pump frequency $\omega_{p}$ then at weak pumping there is a standard Lorentzian response, while at higher power, power broadening
[35] leads to a reduction of the coherent field amplitude near resonance; i.e., there is an antiresonance feature. Turning on hopping, the location of the antiresonance shifts away from the low-power resonance. Eventually it shifts so far that the coherent field amplitude vs pump frequency develops a jump and an associated bistability. Such bistability is analogous to that known in the Dicke model when driving above resonance, where nonlinearity can blueshift the polariton frequency into resonance.

Let us note at this stage that although the existence of bistability is due to the mean-field decoupling, its presence is indicative of physically meaningful bimodal distributions in the true density matrix [36,37]. The equation of motion for the full-system density matrix is linear and so either has a unique steady state or a degenerate subspace of steady states. The mean-field decoupling instead produces a nonlinear equation for the single-site density matrix, which may have multiple distinct solutions - these distinct solutions can thus describe bistability. Where mean-field theory would predict bistability, the full density matrix would generally have a configuration with a significant weight near both of these mean-field solutions, but with a fixed ratio between their weights and a tail of finite probability states that connect these. Both the ratio of weights and the existence of the intermediate states cannot be found by mean-field theories and require consideration of fluctuations, specifically instanton and soliton corrections that would describe tunneling between different mean-field configurations [38]. It is, however, worth noting that all these statements relate to the ensemble averaged steady state of the system. If a system is prepared near to one of the two bistable states, the subsequent dynamics will initially remain near that configuration until a tunneling event causes a transition to the other state. Such tunneling (quantum, thermal, or induced by external noise) can cause transitions in both directions and eventually produces a fixed ratio between the two parts of the bimodal distribution.

Since the spacing of energy levels of the JCHM is anharmonic, in the limit of relatively weak hopping, the problem can be understood quantitatively by restricting the on-site problem to a reduced Hilbert space of 0,1 excitations. As discussed in [28,34], this is valid as long as other excitations are sufficiently far from resonance, $U_{\text {eff }} \gg f$, where $U_{\text {eff }}$ is an effective anharmonicity (which vanishes for large hopping). This reduces the problem to

$$
H_{\mathrm{eff}}=\sum_{i}\left(\frac{\eta}{2} \tau_{i}^{z}+\tilde{f} \tau_{i}^{x}\right)-\frac{\tilde{J}}{z} \sum_{\langle i j\rangle} \tau_{i}^{+} \tau_{j}^{-},
$$

where $\tau_{i}^{\alpha}$ are Pauli matrices in the reduced Hilbert space and the effective parameters are $\eta=(J+\epsilon-$ $\left.\sqrt{(J-\epsilon)^{2}+4 g^{2}}\right) / 2-\omega_{p}, \tilde{J}=J \sin ^{2} \theta$, and $\tilde{f}=-f \sin \theta$ with $\tan (2 \theta)=2 g /(J-\epsilon)$. Losses are described by $\sum_{i}(\tilde{\kappa} / 2) \mathcal{L}\left[\tau_{i}^{-}\right]$with $\tilde{\kappa}=\kappa \sin ^{2} \theta+\gamma \cos ^{2} \theta$. Since $J \ll g$ is assumed one may further approximate $\eta \simeq-g+(J+$ $\epsilon) / 2-\omega_{p}, \theta \simeq \pi / 4$. The steady state of this problem can be reduced to coupled equations for the coherent field amplitude $\psi=\left\langle\tau^{-}\right\rangle$, an effective detuning $\Delta=\eta+\tilde{J}(2 n-1)$, and the 


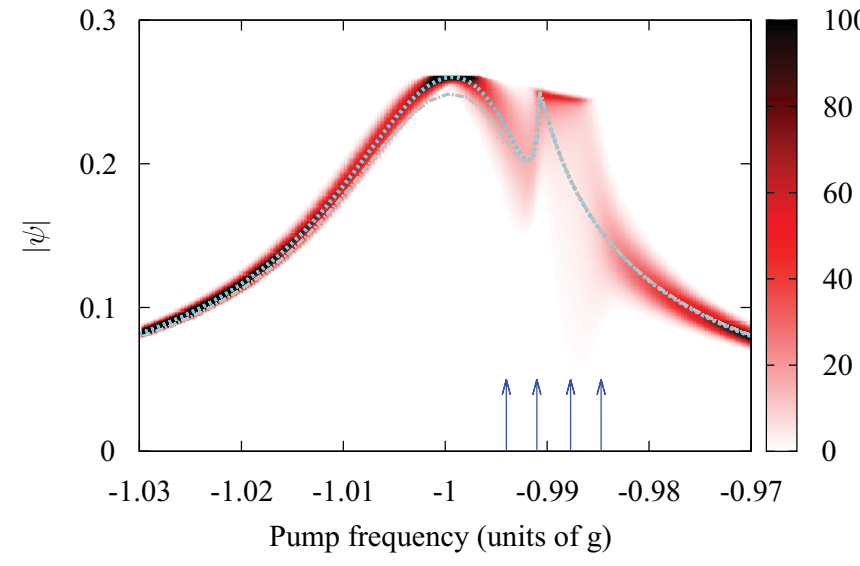

FIG. 1. (Color online) Probability distribution of $|\psi|$ as a function of pump frequency for $J<J_{c}$. The dotted (cyan [light gray]) line indicates the value of $|\psi|$ in the clean limit, and the color map shows the probability distribution of $|\psi|$ for Gaussian disorder of variance $\sigma_{\epsilon} / g=0.002 g$. The dash-dotted (gray) line indicates the approximate solution to the clean case given in Eq. (6). Arrows mark the values of pump frequency at which the full probability distribution of complex $\psi$ is shown in Fig. 2. Other parameters are $f=\kappa=\gamma=0.005 \mathrm{~g}, \mathrm{~J} / \mathrm{g}=0.020$, and a geometry with $z=2$ is assumed.

excited-state population $n=\left\langle 1+\tau^{z}\right\rangle / 2$,

$\psi=\frac{\tilde{f}(\Delta-i \tilde{\kappa} / 2)}{\Delta^{2}+(\tilde{\kappa} / 2)^{2}+2 \tilde{f}^{2}}, \quad n=\frac{\tilde{f}^{2}}{\Delta^{2}+(\tilde{\kappa} / 2)^{2}+2 \tilde{f}^{2}}$.

One may thus see that for $\tilde{f} \ll \tilde{\kappa}$ one has resonance at $\eta=$ $\tilde{J}=J / 2$ giving $\omega_{p} \simeq-g$. In contrast, for larger $\tilde{f}$ one has $n \rightarrow 1 / 2$ and the center of the antiresonance is at $\eta=0$, i.e., $\omega_{p}=-g+J / 2$. Such behavior is already clear in the clean limit shown in Fig. 1, even with $f=\kappa=\gamma$.

For large enough $J$, there are multiple solutions of the above equations. Equivalently this means $\eta$ is a nonmonotonic function of $\Delta$, and so by writing

$$
\eta=\Delta+\tilde{J}-\tilde{J} \frac{2 \tilde{f}^{2}}{\Delta^{2}+(\tilde{\kappa} / 2)^{2}+2 \tilde{f}^{2}}
$$

one can find the critical value of $\tilde{J}$ for bistability by seeking the solution of $d \eta / d \Delta=0=d^{2} \eta / d \Delta^{2}$. This yields

$$
\tilde{J}_{c}=\frac{4}{\tilde{f}^{2}}\left(\frac{2 \tilde{f}^{2}+(\tilde{\kappa} / 2)^{2}}{3}\right)^{3 / 2} .
$$

For $\tilde{J}>\tilde{J}_{c}$, there is a range of $\eta$ (i.e., pump frequencies) for which $\Delta(\eta)$ and thus $\psi(\eta)$ are multivalued and so describe bistability.

\section{B. Effects of disorder}

We consider first the effects of disorder when $J \lesssim J_{c}$, so there is no bistability, but a strong distortion compared to $J=0$. The probability distribution of the amplitude of the coherent field strength, $P(|\psi|)$, in this case is shown in Fig. 1, and cross sections showing the full probability distribution $P(\psi)$ over the complex plane are given in Fig. 2. We consider here parameters as discussed in Ref. [28] for ease

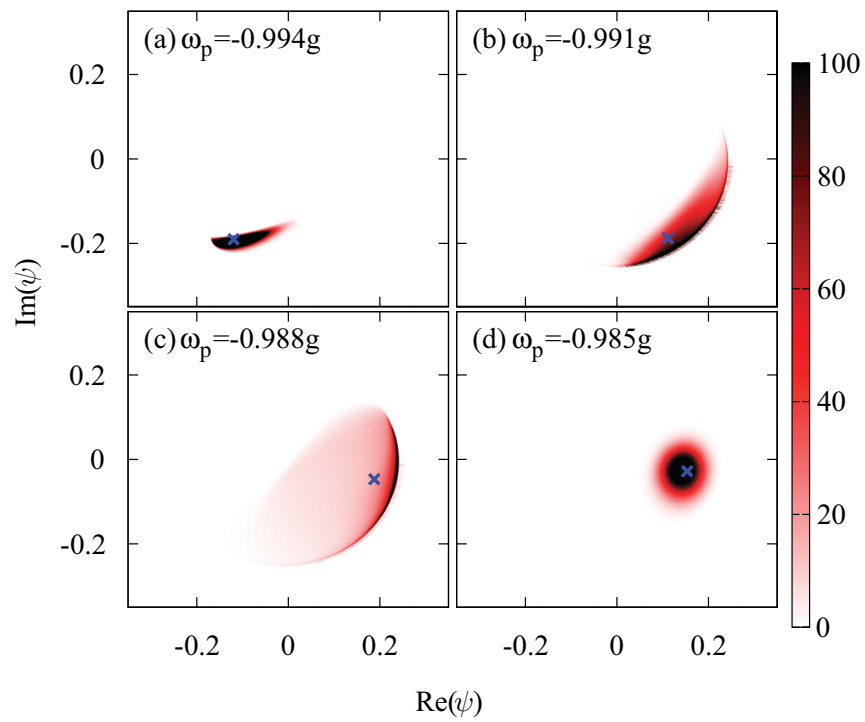

FIG. 2. (Color online) Probability distribution of the complex observable $\psi$ at four different pump frequencies for $J<J_{c}$. The blue (gray) crosses indicate the value of $\psi$ found for the clean case, and the color map shows the probability distribution with $\sigma_{\epsilon} / g=0.002$. All parameters are as in Fig. 1.

of comparison. For the inclusion of disorder, one may note that a typical scale of disorder in recent experiments [39] is $\sigma_{\epsilon} \sim$ $1 \mathrm{MHz}$, corresponding to $0.002 \lesssim \sigma_{\epsilon} / g \lesssim 0.005$. We show results for $\sigma_{\epsilon} / g \simeq 0.002$; larger disorders show very similar behavior.

For most pump frequencies, disorder has a relatively weak effect but near the antiresonance feature it causes a much larger effect. This can easily be understood from the discussion of the clean case above: In this regime $\omega_{p} \simeq-g+J / 2$, and the effective detuning $\Delta_{i} \simeq \eta_{i} \simeq-g+\left(J+\epsilon_{i}\right) / 2-\omega_{p} \simeq \epsilon_{i} / 2$. Thus, near the antiresonance, the variance of $\Delta$ is large compared to its mean value. Since the variance of disorder is of the same order as the linewidth $\kappa$, one finds in this regime that the phase of the on-site order parameter can vary significantly. This is clearly seen in Fig. 2(b). In contrast, away from this point, the mean value of $\Delta$ is much larger than its variance, and so disorder has only a weak effect on the phase and amplitude; hence the clean results are recovered.

As one continues to increase the pump frequency above the antiresonance, the field amplitude remains notably higher than in the clean case, and [as seen in Fig. 2(c)] the phase distribution remains broad. The increased amplitude can be clearly understood as an effect of the phase distribution: Increasing the phase distribution means the convolution distribution $Q(\phi)$ moves toward smaller $\phi$. Since the field seen by a given site is given by $f^{\text {eff }}=f-J \phi / z$, and since $\operatorname{Re}(\phi)>0$, reducing $|\phi|$ increases the effective driving and thus increases the amplitude.

The phase spreading seen here signifies an important distinction between the thermal and the nonequilibrium disordered problem. In the thermal case, a real distribution of $\psi$ is stable, but in the nonequilibrium case there is always a distribution of phase and near resonance this becomes particularly notable. In the current case, phase symmetry is broken by the external pump. However, for incoherent 


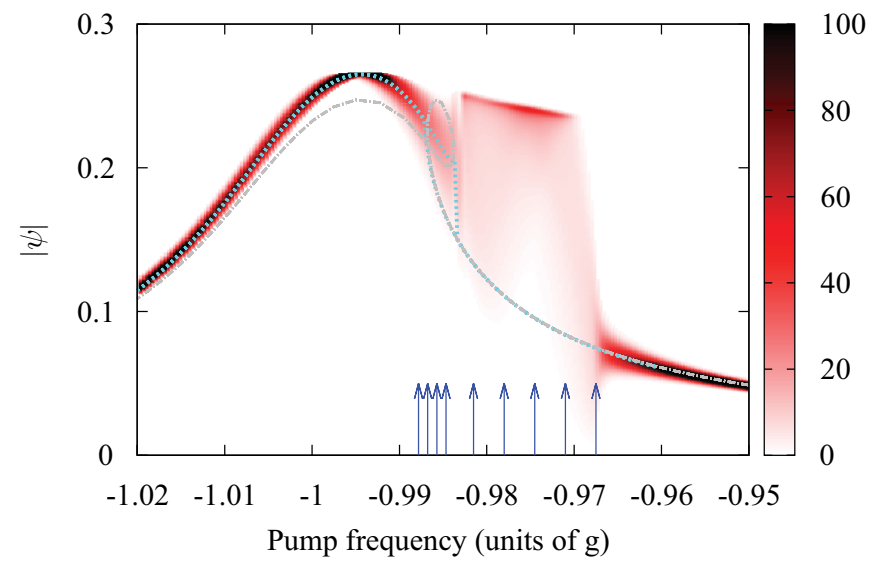

FIG. 3. (Color online) Probability distribution of $|\psi|$ as a function of pump frequency for $J>J_{c}$. Lines and parameters are as for Fig. 1, except $J / g=0.04$ in this case.

pumping, phase symmetry is not broken. The presence of phase spreading then means that following Imry and Ma [40], no spontaneous phase symmetry breaking is possible in $d<4$. This is quite different from the equilibrium JCHM where a superfluid (super-radiant) state with phase symmetry breaking is expected in $d>2$. A similar observation has recently been made for the disordered polariton condensate [41].

As discussed above, in the clean case, for $J>J_{c}$ bistability occurs because of the multivalued nature of $\Delta(\eta)$. However, the range of detunings where this occurs is the same range where strong phase spreading was seen, and thus disorder strongly affects the behavior in exactly this region. Thus, as seen in Fig. 3, the disordered case with a typical disorder strength $\sigma_{\epsilon} / g=0.002$ does not show any bistability, with no weight near the new clean solution which appears as $\omega_{p}$ is increased. The absence of bistability is revealed by noting that the same steady state is found independent of starting distribution of $P(\psi)$; in the current case this was tested by comparing a

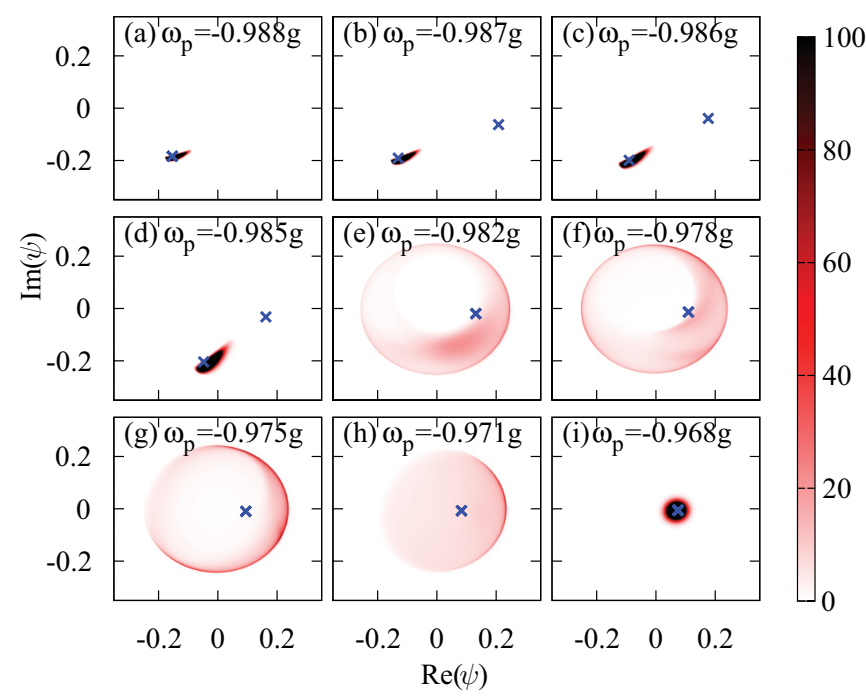

FIG. 4. (Color online) Probability distribution of the complex observable $\psi$ at nine different pump frequencies for $J>J_{c}$. All parameters are as in Fig. 3. "sweep" of slowly increasing or decreasing pump frequency; both cases lead to identical results. Since a unique solution exists in these cases, this may be taken as an approximation of the true asymptotic state of the disordered system. One may also note in Figs. 4(e)-4(h) that even once the low $\omega_{p}$ solution vanishes, weight is not concentrated near the high $\omega_{p}$ solution until significantly above the antiresonance frequency, as there is a strong effect of the phase distribution.

\section{CONCLUSIONS AND OPEN QUESTIONS}

We have presented a nonequilibrium extension of stochastic mean-field theory, applicable to problems of coupled cavities with rather general forms of driving and dissipation. Using this approach we studied the effect of disorder on the driven dissipative JCHM. Near the antiresonance, disorder introduces significant phase spreading, which in turn increases the coherent field amplitude over a range of pump frequencies above the antiresonance.

The results presented above for the dissipative driven JCHM clearly demonstrate that the combination of open quantum systems with disorder can lead to behavior that is not seen with only one of these two ingredients in isolation. Such behavior prompts an important question regarding whether dissipative coupled matter-light systems could ever be used as "quantum simulators" of disordered models. At the same time, it indicates that there are open questions as to what the phase diagram of incoherently pumped disordered dissipative systems may be. In some cases, such as the Rabi-Hubbard model [26], only discrete symmetries exist and so the effects of disorder should not destroy the symmetry-broken phase, and the behavior may be equivalent to that of the site-disordered transverse-field Ising model. However, for cases with continuous symmetry it is unclear whether any phase boundaries exist, since neither the superfluid nor Mott-insulating states survive the effects of dissipation. Such questions can be in part addressed by the SMFT approach described here.

Another challenge is to go beyond the stochastic mean-field limit presented here and produce alternate methods to treat open disordered lattice problems. As with all mean-field approaches, SMFT neglects quantum correlations between different sites, an assumption only valid in the limit of high coordination. Mean-field theory in general neglects quantum nonlinearities. In the current problem the mean-field decoupling is only applied to the coupling between different cavities; thus for $J \rightarrow 0$ it reproduces the exact results [34] of the single-site problem. However, with increasing $J$ mean-field theory introduces an approximation by neglecting quantum correlations between sites. In addition to the limitations of mean-field decouplings, SMFT makes a second assumption, that there is no correlation between the on-site energy and the field distribution. Such an assumption implies self-averaging, while it is known that self-averaging breaks down in the equilibrium Bose glass [23]. An alternative approach that may circumvent this is to consider extensions of the cavity method, e.g., Ref. [42]. For the purpose of understanding the behavior of currently achievable experiments [7], finite-size simulations of the mean-field [28] or beyond-mean-field [29,30] dynamics may be more appropriate. However, a full understanding of the behavior of such dissipative models may well depend on 
rare events, not captured in finite-size simulations, so methods such as that presented here may play an important role.

In conclusion, the combination of dissipation and disorder can lead to types of behavior in coupled cavity arrays that cannot be seen in either the clean nonequilibrium system or disordered equilibrium case. This suggests that such cavity arrays may not be appropriate as quantum simulators to understand equilibrium disordered problems. Stochastic mean-field theory can provide a simple route to address some classes of system but leads to questions that require more sophisticated approaches to nonequilibrium disordered problems.

\section{ACKNOWLEDGMENTS}

G.K. acknowledges support from the Nuffield trust and University of St Andrews URIP program. F.B.F.N. acknowledges support from EPSRC. J.K. and F.B.F.N. acknowledge discussions with H. Türeci and S. Schmidt. J.K. acknowledges useful discussions with M. Schiró, G. Biroli, and P. Phillips; hospitality from KITP Santa Barbara; and financial support from the EPSRC program "TOPNES" (EP/I031014/1) and EPSRC (EP/G004714/2). This research was supported in part by the National Science Foundation under Grant No. NSF PHY11-25915.
[1] S. Lloyd, Science 273, 1073 (1996).

[2] I. Bloch, J. Dalibard, and W. Zwerger, Rev. Mod. Phys. 80, 885 (2008).

[3] H. Weimer, M. Müller, I. Lesanovsky, P. Zoller, and H. P. Büchler, Nat. Phys. 6, 382 (2010).

[4] B. P. Lanyon, C. Hempel, D. Nigg, M. Müller, R. Gerritsma, F. Zähringer, P. Schindler, J. T. Barreiro, M. Rambach, G. Kirchmair, M. Hennrich, P. Zoller, R. Blatt, and C. F. Roos, Science 334, 57 (2011).

[5] J. T. Barreiro, M. Müller, P. Schindler, D. Nigg, T. Monz, M. Chwalla, M. Hennrich, C. F. Roos, P. Zoller, and R. Blatt, Nature (London) 470, 486 (2011).

[6] J. Koch and K. Le Hur, Phys. Rev. A 80, 023811 (2009).

[7] A. A. Houck, H. E. Türeci, and J. Koch, Nat. Phys. 8, 292 (2012).

[8] S. Schmidt and J. Koch, arXiv:1212.2070.

[9] F. Dimer, B. Estienne, A. S. Parkins, and H. J. Carmichael, Phys. Rev. A 75, 013804 (2007).

[10] K. Baumann, C. Guerlin, F. Brennecke, and T. Esslinger, Nature (London) 464, 1301 (2010).

[11] D. Nagy, G. Szirmai, and P. Domokos, Phys. Rev. A 84, 043637 (2011).

[12] M. J. Bhaseen, J. Mayoh, B. D. Simons, and J. Keeling, Phys. Rev. A 85, 013817 (2012).

[13] B. Öztop, M. Bordyuh, O. E. Müstecaplolu, and H. E. Türeci, New J. Phys. 14, 085011 (2012).

[14] E. G. D. Torre, S. Diehl, M. D. Lukin, and P. Strack, arXiv: $1210.3623 \mathrm{v} 1$.

[15] M. J. Hartmann, F. G. S. L. Brandão, and M. B. B. Plenio, Laser Photon. Rev. 2, 527 (2008).

[16] S. Trotzky, L. Pollet, F. Gerbier, U. Schnorrberger, I. Bloch, N. V. Prokofev, B. Svistunov, and M. Troyer, Nat. Phys. 6, 998 (2010).

[17] M. P. A. Fisher, P. B. Weichman, G. Grinstein, and D. S. Fisher, Phys. Rev. B 40, 546 (1989).

[18] J. E. Lye, L. Fallani, M. Modugno, D. S. Wiersma, C. Fort, and M. Inguscio, Phys. Rev. Lett. 95, 070401 (2005).

[19] D. Clément, P. Bouyer, A. Aspect, and L. Sanchez-Palencia, Phys. Rev. A 77, 033631 (2008).

[20] J. Billy, V. Josse, Z. Zuo, A. Bernard, B. Hambrecht, P. Lugan, D. Clément, L. Sanchez-Palencia, P. Bouyer, and A. Aspect, Nature (London) 453, 891 (2008).
[21] M. White, M. Pasienski, D. McKay, S. Q. Zhou, D. Ceperley, and B. DeMarco, Phys. Rev. Lett. 102, 055301 (2009).

[22] V. Gurarie, L. Pollet, N. V. Prokofev, B. V. Svistunov, and M. Troyer, Phys. Rev. B 80, 214519 (2009).

[23] F. Krüger, S. Hong, and P. Phillips, Phys. Rev. B 84, 115118 (2011).

[24] A. D. Greentree, C. Tahan, J. H. Cole, and L. C. L. Hollenberg, Nat. Phys. 2, 856 (2006).

[25] K. L. Hur, in Understanding Quantum Phase Transitions, edited by L. D. Carr (Taylor and Francis, Boca Raton, 2010).

[26] M. Schiró, M. Bordyuh, B. Öztop, and H. E. Türeci, Phys. Rev. Lett. 109, 053601 (2012); H. Zhang and Y. Takeda, Phys. Rev. A 84, 043819 (2011).

[27] E. Mascarenhas, L. Heaney, M. C. O. Aguiar, and M. F. Santos, New J. Phys. 14, 043033 (2012).

[28] F. Nissen, S. Schmidt, M. Biondi, G. Blatter, H. E. Türeci, and J. Keeling, Phys. Rev. Lett. 108, 233603 (2012).

[29] T. Grujic, S. R. Clark, D. Jaksch, and D. G. Angelakis, New J. Phys. 14, 103025 (2012).

[30] T. Grujic, S. R. Clark, D. Jaksch, and D. G. Angelakis, arXiv:1212.3012.

[31] H. Weber and M. Vojta, Eur. Phys. J. B 53, 185 (2006).

[32] U. Bissbort and W. Hofstetter, Europhys. Lett. 86, 50007 (2009).

[33] U. Bissbort, R. Thomale, and W. Hofstetter, Phys. Rev. A 81, 063643 (2010).

[34] L. S. Bishop, J. M. Chow, J. Koch, A. A. Houck, M. H. Devoret, E. Thuneberg, S. M. Girvin, and R. J. Schoelkopf, Nat. Phys. 5, 105 (2008).

[35] D. F. Walls and G. J. Milburn, Quantum Optics, 2nd ed. (Springer, Berlin, 2008).

[36] P. Drummond and D. Walls, J. Phys. A.: Math. Gen. 13, 725 (1980).

[37] L. Lugiato, Prog. Opt. 21, 69 (1984).

[38] A. Kamenev, Field Theory of Non-equilibrium Systems (Cambridge University Press, Cambridge, 2011).

[39] D. L. Underwood, W. E. Shanks, J. Koch, and A. A. Houck, Phys. Rev. A 86, 023837 (2012).

[40] Y. Imry and S.-K. Ma, Phys. Rev. Lett. 35, 1399 (1975).

[41] A. Janot, T. Hyart, B. Rosenow, and P. R. Eastham (unpublished).

[42] G. Semerjian, M. Tarzia, and F. Zamponi, Phys. Rev. B 80, 014524 (2009). 